\title{
A Solitary Fibrous Tumor of the Subglottic Larynx: Case Report and Literature Review
}

\author{
Sung-Joon Park ${ }^{1}$, Young Hen Lee ${ }^{1}$, Ki Yeol Lee ${ }^{1}$, Kyoung Ho Oh', Younghye Kim \\ ${ }^{1}$ Department of Radiology, Korea University Ansan Hospital, Ansan, Korea \\ ${ }^{2}$ Department of Otorhinolaryngology-Head and Neck Surgery, Korea University Ansan Hospital, Ansan, Korea \\ ${ }^{3}$ Department of Pathology, Korea University Ansan Hospital, Ansan, Korea
}

Background: Solitary fibrous tumors (SFT) arising from the larynx are extremely rare; most occur in the supraglottic larynx.

Case Report: Herein, we detail a new case of a subglottic SFT presenting as a well-encapsulated soft-tissue tumor with hoarseness. It showed isoattenuation, similar to the adjacent muscle on pre-contrast computed tomography (CT) images, and strong, heterogeneous enhancement following contrast material administration, which may reflect hypervascularity. On pathological examination, it consisted of spindle cells, squeezed between abundant collagen, and focally prominent vascularity in a staghorn feature. The tumor cells were immu- noreactive for $C D 34, B C L 2$ and $C D 99$. Based on the combination of architectural and immunohistochemical criteria, we ultimately diagnosed this case as an SFT.

Conclusion: Until now, only 14 cases of laryngeal SFT have been described in the literature: 13 located in the supraglottic areas and only one located in the subglottic/tracheal area. Therefore the present case is the second case of a subglottic SFT reported. Although rare, SFT should be included in the differential diagnosis of a well-marginated laryngeal mass with highly intense contrast enhancement, which suggests rich tumor vascularity.

Keywords: Larynx, benign neoplasm, solitary fibrous tumor
The solitary fibrous tumor (SFT) is an uncommon spindle cell neoplasm, described initially as a pleural-based lesion. SFT have also been found in numerous extrapleural sites, including the extracranial head and neck regions (1-3) . Moreover, SFTs arising from the larynx are extremely rare; most have occurred in the supraglottic larynx (4-6) . Herein, we report a new case of subglottic SFT and provide a review of the relevant literature.

\section{CASE PRESENTATION}

A 33-year-old non-smoking man presented to the Ear, Nose and Throat (ENT) clinic with a 3-month history of hoarseness. He had no history of recent upper respiratory infections or palpable neck lymph nodes. An endoscopic examination revealed a round submucosal mass with greatest diameter of 15 $\mathrm{mm}$, covered by prominent vessels, on the anterior surface of the subglottic larynx, leading to luminal narrowing (Figure 1). The normal appearance of the supraglottis and glottis was noted, along with the preservation of vocal cord motility.

The patient underwent neck computed tomography (CT) (Ingenuity Core 128, Philips Healthcare; Best, the Netherlands) with contrast administration for evaluation of the mass lesion. This $1.3 \times 1.0 \times 1.3 \mathrm{~cm}$ well-demarcated lesion showed isoattenuation relative to the adjacent muscle on a pre-contrast CT with marked heterogeneous enhancement on contrast-enhanced CT images obtained by a 40 -second scan delay. However, no evidence of adjacent cartilage destruction, transglottic/tracheal extension or neck lymph node enlargement was observed (Figure 2). Based on the tumor's location and characteristics, the preoperative radiologic differential diagnosis included a hemangioma, hemangiopericytoma, paraganglioma and, less likely, intralaryngeal ectopic thyroid tissue. 


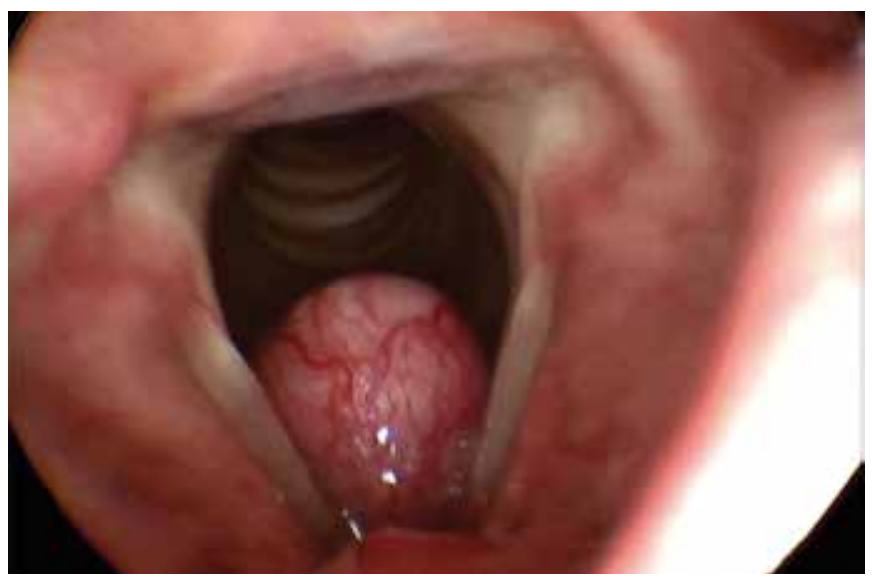

FIG. 1. On endoscopic examination, a round, well-demarcated lesion was noted midline at the level of the subglottis. The lesion was covered by dilated vessels.

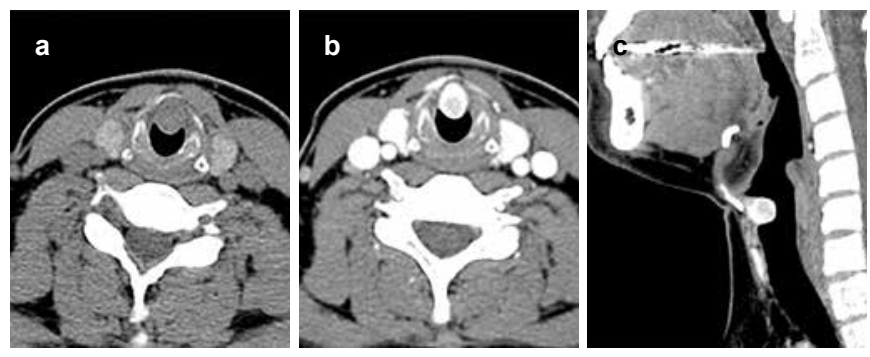

FIG. 2. a-c. Pre-contrast and contrast-enhanced CT images demonstrated a $1.3 \times 1.0 \times 1.3 \mathrm{~cm}$ round-shaped tumor located at the subglottic larynx without transglottic or extralaryngeal extension. Its density was similar to that of adjacent muscle on pre-contrast image (a) and became markedly increased, like that of the thyroid gland, on the contrast-enhanced images with a 40 second scan delay $(b, c)$.
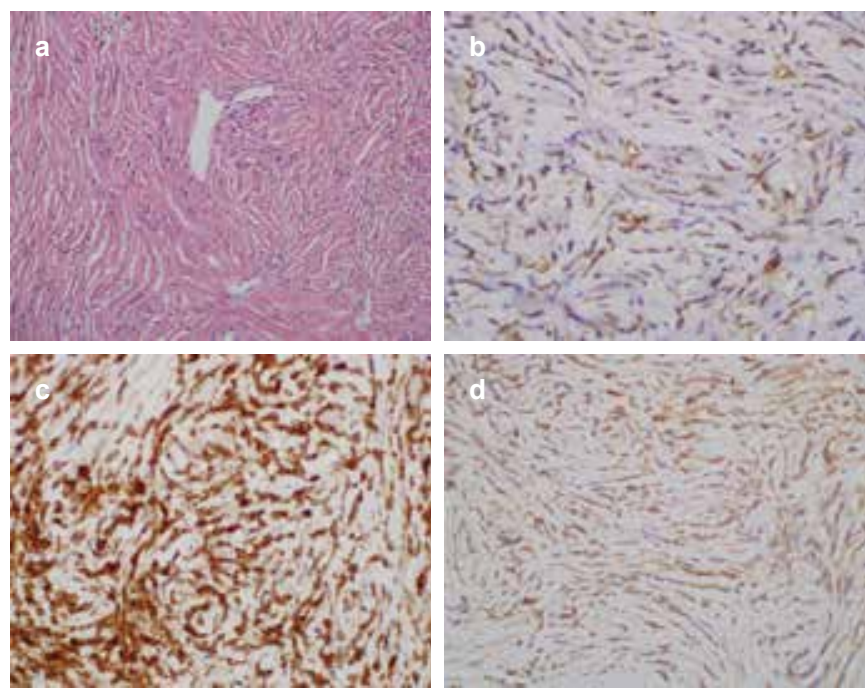

FIG. 3. a-d. The histologic examination showed randomly arranged spindle cells separated by abundant collagen. Staghorn-shaped blood vessels were also found (a, H\&E x200). Immunohistochemically, the tumor cells were positive for CD34 (b), BCL2 (c), and they showed diffuse positivity for CD99 (d).
We decided to excise this lesion and conducted laser laryngeal microsurgery using a suspension laryngoscope. The tumor was located under the anterior commissure. The tumor was retracted with microforceps and resected with a $\mathrm{CO}_{2}$ laser. It was a whitish mass. Bleeding was minimal during the operation.

On pathological examination, the tumor showed no pattern, consisting rather of spindle cells, squeezed in between abundant collagen (Figure 3). Focally prominent staghorn vessels are found. Artifactual cracks were present between cells and collagen and between collagen bundles. On immunohistochemical staining, the tumor cells were characteristically immunoreactive for $C D 34$ and $B C L 2$. They also showed diffuse positivity for $C D 99$. Mitotic figure was rarely detected. There was no necrosis or cellular atypia. We finally diagnosed this case as SFT.

Written informed consent was obtained from the patient.

\section{DISCUSSION}

Although approximately $50 \%$ of SFTs occur in the thorax, they rarely arise in extrathoracic spaces, including the liver, pancreas, spleen, retroperitoneum and extracranial head and neck regions, such as the oral cavity, orbit, nasal cavity, parapharyngeal space, infratemporal fossa and others (1-3). SFTs arising from head and neck region are rare as only $6 \%$ of the SFT cases, and the oral cavity is the most frequent subsite affected. Moreover, SFT arising from the larynx are extremely rare. Until now, only 14 cases of laryngeal SFT have been described in the literature, 13 located in the supraglottic areas (4-6), and only one located in the subglottic/tracheal area (7), therefore the present case is second case of a subglottic SFT reported. Most SFTs occur in middleaged adults, presenting as slow-growing painless masses with a variable degree of obstructive symptoms. Hoarseness is a common presentation for tumors of the larynx, and symptoms of sore throat, dysphagia, and referred otalgia may also be presented. Although complete resection is recommended to prevent local recurrence, SFTs tend to behave in a benign fashion $(3,4)$.

Usually, SFTs appear on CT and MRI as intensely contrastenhanced, polypoid masses protruding into, and partially to completely occluding, the laryngeal lumen. CT and MRI images can reflect their rich vascularity. In addition, less enhancing areas of SFT pathologically correlate with myxoid or cystic degeneration (2), so the central low attenuated portion seen in our case is presumed to represent the less cellular stromal area. Their signal intensities are generally intermediate on T1-weighted images and heterogeneously hyperintense on T2-weighted images $(1,4,5)$. On dual-phased CT and dynamic contrast-enhanced MRI, SFTs demonstrate rapid, intense enhancement during early-phase scanning, followed by a progressive washout of contrast material during delayed 
scanning, which may suggest the possibility of SFTs $(1,2)$. At our institution, a contrast-enhanced CT examination was performed for the neck lesion on a 128-row multidetector CT scanner with a 40-second scan delay, consistent with the early-phase scanning defined by the previous study (1). Thus, the rapid, intense enhancement of the SFT was reconfirmed through our contrast-enhanced CT image.

Microscopically, SFTs exhibit patternless architecture consisting of a varying number of spindle cells, randomly arranged on a collagenous background with variable vascularity. Typically, histological features associated with aggressive behavior including nuclear atypia, markedly increased cellularity, areas of necrosis and greater than 4 mitoses per 10 HPF, are rarely encountered in SFTs. The SFT diagnosis is supported by a characteristic immunohistochemical profile. $C D 34$, a kind of myofibroblastic marker, is considered to be a characteristic marker to diagnose SFT. In addition to CD34, SFTs are also positive for mesenchymal markers (vimentin), and in about $50 \%$ of cases, for CD99 and BCL-2 protein, but they are consistently negative for cytokeratin, alpha-SMA, S-100, and CD31 (3).

Despite its characteristic immunohistochemical features, SFT of the head and neck remains a diagnostic challenge to both clinicians and radiologists. The imaging characteristics of SFT include the well-demarcated appearance of a submucosal tumor with strong enhancement on CT and MRI, typical of nonsquamous cell tumors, which account for $2-5 \%$ of all laryngeal tumors. These lesions include hemangiomas, hemangiopericytomas, paragangliomas, soft tissue sarcomas and, more rarely, SFT, as in our case (8). However, it seems to be difficult to differentiate SFT from hemangioma or hemangiopericytoma based on the clinico-radiologic appearance alone (8).

Most SFTs show a benign clinical course, but approximately $15-20 \%$ of SFT exhibit more aggressive behavior with tumor recurrences and/or distant metastases. General treatment choice of SFT is surgical resection with negative margins, but there is no effective treatment option for metastatic or advanced disease of SFT $(3,4)$. Long-term follow up is important due to malignant potential of SFT even after radical resection. In terms of the histopathology, some authors report low expression or loss of CD34 and high IGF2 showed a higher statistical correlation to the malignant potential in SFTs than the mitotic index (9).

In conclusion, we presented a rare case of subglottic SFT occurring in a young adult non-smoker. The tumor manifested as a well-demarcated submucosal mass with strong contrastenhancement on CT images, which reflected hypervascularity. This hypervasculatiry was then confirmed by immunoreactivity for $C D 34, B C L 2$ and $C D 99$. To our knowledge, this is the second report of a subglottic SFT. Although rare, SFT should be included in the differential diagnosis of a laryngeal submu- cosal mass with highly intense contrast enhancement, which indicates rich tumor vascularity.

\section{Ethics Committee Approval: N/A.}

Informed Consent: Written informed consent was obtained from the patient who participated in this case.

Peer-review: Externally peer-reviewed.

Author contributions: Concept - S.J.P., Y.H.L.; Design - S.J.P., Y.H.L.; Supervision - K.Y.L.; Resource - K.H.O.; Materials - K.H.O, Y.K.; Data Collection and/or Processing - S.J.P., K.H.O., Y.K.; Analysis and/or Interpretation - S.J.P., Y.K.; Literature Search - S.J.P., Y.H.L.; Writing - S.J.P., Y.H.L.; Critical Reviews - Y.H.L.

Conflict of Interest: No conflict of interest was declared by the authors.

Financial Disclosure: The authors declared that this case has received no financial support.

\section{REFERENCES}

1. Liu Y, Li K, Shi H, Tao X. Solitary fibrous tumours in the extracranial head and neck region: correlation of $\mathrm{CT}$ and MR features with pathologic findings. Radiol Med 2014;119:910-9. [CrossRef]

2. Kim HJ, Kim HJ, Kim YD, Yim YJ, Kim ST, Jeon P, et al. Solitary fibrous tumor of the orbit: CT and MR imaging findings. AJNR Am J Neuroradiol 2008;29:857-62. [CrossRef]

3. Ganly I, Patel SG, Stambuk HE, Coleman M, Ghossein R, Carlson D, et al. Solitary fibrous tumors of the head and neck: a clinicopathologic and radiologic review. Arch Otolaryngol Head Neck Surg 2006;132:517-25. [CrossRef]

4. Morvan JB, Veyrieres JB, Mimouni O, N'Guyen AT, Fouet $\mathrm{B}$, Cathelinaud O, et al. Solitary fibrous tumour of the larynx: a case report. Eur Ann Otorhinolaryngol Head Neck Dis 2011;128:262-5. [CrossRef]

5. Chang SK, Yoon DY, Choi CS, Yun EJ, Seo YL, Nam ES. CT, $\mathrm{MR}$, and angiography findings of a solitary fibrous tumor of the larynx: a case report. Korean J Radiol 2008;9:568-71. [CrossRef]

6. Fábio Muradás Girardi1 MBB, Adriana Muradás Girardi. Solitary fibrous tumor of the larynx: report of two new cases. J Bras Patol Med Lab 2014;50:229-33.

7. Thompson LD, Karamurzin Y, Wu ML, Kim JH. Solitary fibrous tumor of the larynx. Head Neck Pathol 2008;2:67-74. [CrossRef]

8. Razek AA, Huang BY. Soft tissue tumors of the head and neck: imaging-based review of the WHO classification. Radiographics 2011;31:1923-54. [CrossRef]

9. Schulz B, Altendorf-Hofmann A, Kirchner T, Katenkamp D, Petersen I, Knösel T. Loss of cd34 and high igf2 are associated with malignant transformation in solitary fibrous tumors. Pathol Res Pract 2014;210:92-7. [CrossRef] 